\title{
WHITEHEAD GROUPS OF FINITE GROUPS ${ }^{1}$
}

\author{
BY MICHAEL R. STEIN
}

In 1966, Milnor surveyed in this Bulletin [23] the concept of Whitehead torsion, focusing on the definition, topological significance and computation of Whitehead groups and their relationship to algebraic $K$-theory and the congruence subgroup problem. As Milnor showed in that survey [23, Appendix 1], an affirmative solution to the congruence subgroup problem for algebraic number fields would imply that for any finite abelian group $G$, $S K_{1}(\mathrm{Z} G)=0$; i.e. that the Whitehead group of a finite abelian group $G$ is torsion-free. At that time the status of the congruence subgroup problem was uncertain [23, pp. 360, 416]; it was subsequently shown to have a negative solution by Bass, Milnor and Serre [7]. Nevertheless, until 1972 all finite abelian groups for which computations could be made had trivial $S K_{1}$ (cf. [5, p. 624]) and the question of whether these groups could be nontrivial remained open [6].

An intensive study of Milnor's $K_{2}$-functor on discrete valuation rings [10] and the application of Mayer-Vietoris sequences in algebraic $K$-theory led to the first examples of finite abelian groups with nontrivial $S K_{1}$ and have provided an algorithm for the computation of such $S K_{1}$ 's in general. In addition, the first steps towards the computation of $S K_{1}(\mathrm{Z} G)$ for nonabelian finite groups have been taken by several authors.

It is my purpose to survey these techniques and computations, beginning where Milnor left off in 1966. I will rely heavily on his article for background material; all unexplained notations and terminology should be sought there.

If $G$ is a finite group, its order is denoted $|G|$ and its abelianization, $G^{\text {ab }}$. A finite field with $q$ elements is denoted $\mathbf{F}_{q}$. The units of a ring $A$ are denoted $A^{*}$ or $U(A)$.

I would like to thank Bruce Magurn, Keith Dennis and Michael Keating for their helpful comments.

1. Whitehead groups. Let $R$ be an associative ring with 1 , and suppose $n \geqslant 1$. Let $G L_{n}(R)$ be the group of all invertible $n \times n$ matrices with entries in $R$. We can embed $G L_{n}(R)$ in $G L_{n+1}(R)$ by sending an $n \times n$ matrix $A$ to $\left(\begin{array}{cc}A & 0 \\ 0 & 1\end{array}\right) \in G L_{n+1}(R)$. This yields homomorphisms $G L_{1}(R) \rightarrow G L_{2}(R) \rightarrow \ldots$; their direct limit is denoted $G L(R)$.

An $n \times n$ matrix is called elementary if it differs from the identity by a single off-diagonal entry. The subgroup generated by all elementary matrices is denoted $E(R)$, and J. H. C. Whitehead proved that $E(R)$ is precisely the

This is an expanded version of an invited address presented at the 745th meeting of the American Mathematical Society, Evanston, Illinois, April 15, 1977; received by the editors September 12, 1977.

AMS (MOS) subject classifications (1970). Primary 18F25, 16A54; Secondary 57C10.

1 This work was partially supported by a grant from the National Science Foundation. 
commutator subgroup of $G L(R)$. We define $K_{1}(R)=G L(R) / E(R)=$ $G L(R)^{\mathrm{ab}}$.

If the ring $R$ is commutative, similar considerations apply to the matrices of determinant 1, and lead to the definition $S K_{1}(R)=S L(R) / E(R)$. In this case there is a direct sum decomposition

$$
K_{1}(R) \approx U(R) \oplus S K_{1}(R),
$$

where $U(R)$ denotes the group of units of $R$, since the inclusion $U(R)=$ $G L_{1}(R) \subset G L(R)$ splits the determinant homomorphism $G L(R) \rightarrow U(R)$ whose kernel is $S L(R)$.

If $I$ is a (two-sided) ideal in $R$, we let $G L(R, I)=\operatorname{ker}(G L(R) \rightarrow G L(R / I))$ and $E(R, I)$ be the normal subgroup of $E(R)$ generated by matrices with all off-diagonal entries in $I$. The relative group $K_{1}(R, I)=G L(R, I) / E(R, I)$ fits into an exact sequence

$$
K_{1}(R, I) \rightarrow K_{1}(R) \rightarrow K_{1}(R / I)
$$

when $R$ is commutative, similar definitions and remarks apply to $S K_{1}(R, I)$ as well.

The following theorem will be crucial to our calculations.

(1.2) Theorem [7, Corollary 4.3]. Let $\mathcal{D}$ be the ring of integers in an algebraic number field $F$ and let a be an ideal of $\mathcal{D}$. Then $S K_{1}(\mathfrak{D}, \mathfrak{a})$ is canonically isomorphic to a cyclic subgroup of order l of the roots of unity, $\mu(F)$, of $F$, where $l=1$ if $\mathfrak{a}=\mathfrak{D}$, or $\mathfrak{a}=(0)$, or $\mathfrak{D}$ is not totally imaginary; and

$$
\operatorname{ord}_{p}(l)=\min _{\mathfrak{p} \mid p}\left[\frac{\operatorname{ord}_{\mathfrak{p}}(\mathfrak{a})}{\operatorname{ord}_{\mathfrak{p}}(p)}-\frac{1}{p-1}\right]_{[0, s]},
$$

where $s=\operatorname{ord}_{p}(\mu(F))$, otherwise.

Here $[x]_{[0, s]}, x \in \mathbf{R}, s \in \mathbf{Z}$, denotes the nearest integer is the interval $[0, s]$ to the largest integer $\leqslant x$.

Let $A$ be any commutative ring and $G$ a finite group. The inclusion of $A$ into the group ring $A G$ splits the augmentation $A G \rightarrow A$ and shows that $K_{1}(A)$ occurs as a direct summand of $K_{1}(A G)$. Also, the composite

$$
G \rightarrow U(A G)=G L_{1}(A G) \rightarrow G L(A G) \rightarrow K_{1}(A G)
$$

induces a homomorphism $G^{\mathrm{ab}} \rightarrow K_{1}(A G)$ which is injective because the composition

$$
G^{\mathrm{ab}} \rightarrow K_{1}(A G) \rightarrow K_{1}\left(A G^{\mathrm{ab}}\right) \stackrel{\text { det }}{\rightarrow} U\left(A G^{\mathrm{ab}}\right)
$$

is just the usual inclusion. We define the Whitehead group, $\mathrm{Wh}(A G)$, to be the quotient of $K_{1}(A G)$ by $K_{1}(A) \oplus G^{\text {ab }}$. When $A=\mathbf{Z}$, we write $\operatorname{Wh}(G)$ for $\mathrm{Wh}(\mathrm{ZG})$. Bass has shown [4] that $\mathrm{Wh}(G)$ is a finitely generated abelian group of rank $r(G)-q(G)$, where $r(G)$ (resp. $q(G)$ ) denotes the number of irreducible real (resp. rational) representations of $G$.

Note that when $G$ is finite abelian, we have the decomposition (1.3) $\mathrm{Wh}(A G) \approx\left(S K_{1}(A G) / S K_{1}(A)\right) \oplus(U(A G) /(U(A) \oplus G))$.

When $A$ is the ring of integers in an algebraic number field, $\operatorname{SK}_{1}(A)=0$ by 
Theorem 1.2 and we have $W h(A G) \approx S K_{1}(A G) \oplus U(A G) /\left(A^{*} \oplus G\right)$. In this case Higman [13] has shown that $A^{*} \oplus G$ contains all the units of finite order in $A G$, so the summand $U(A G) /\left(A^{*} \oplus G\right)$ is torsion-free. We shall see later that $S K_{1}(A G)$ is finite.

2. Group rings of finite abelian groups; some easy examples. Let $F$ be a finite extension field of $Q, G$ a finite abelian group, and $\chi: G \rightarrow C^{*}$ an irreducible character of $G$. Then $\chi(G)$ is a finite cyclic group of roots of unity of order $m$ dividing $|G|$. Let $\bar{\chi}$ be a primitive $m$ th root of unity. Then $\chi$ induces a surjective homomorphism, which we shall also denote $\chi$, from the group algebra $F G$ to $F(\bar{\chi})$. Since $F G$ is semisimple (Maschke's theorem), $\chi$ must be split and $F(\bar{\chi})$ occurs as a simple component of $F G$. If $\mathcal{O}$ is the ring of integers in $F$, the restriction to $\mathscr{D}$ of $\chi$ has image $\mathscr{D}[\bar{\chi}]$.

Conversely, every simple component of $F G$ affords an irreducible representation, and thus an irreducible character, of $G$. This means that every simple component of $F G$ arises in the way described above.

It is possible, however, for distinct irreducible characters of $G$ to give rise to the same simple component of $F G$. For example, if $F=\mathbf{Q}$, two characters $\chi_{1}$, $\chi_{2}$ of $G$ will give rise to the same simple component of $Q G$ if and only if their kernels are the same. The relation " $\chi_{1} \sim \chi_{2}$ if the simple components of $F G$ they give rise to are the same" is an equivalence relation on the set of irreducible characters of $G$; we shall denote by $S_{F}(G)$ (or simply $S$ when $F$ and $G$ are understood) a set of representatives for the equivalence classes under this relation. When $F=\mathbf{Q}, S_{\mathbf{Q}}(G)$ may be taken to be the set of irreducible rational characters of $G$.

It follows from the preceding discussion that for any finite abelian group $G$, there is an isomorphism

$$
\alpha: F G \stackrel{\approx}{\rightarrow} \prod_{x \in S_{F}(G)} F(\bar{\chi})
$$

where $\alpha=\Pi_{X \in S_{F}(G)} \chi$.

Let us write $B=\Pi_{\left.x \in S_{\mathrm{Q}} G\right)} \mathbf{Z}[\bar{\chi}]$. The restriction to $\mathbf{Z} G$ of $\alpha$ is an injective homomorphism $\mathbf{Z G \rightarrow B}$. The conductor, $\mathrm{c}$, from $B$ to $\mathbf{Z} G$ is the largest ideal of $B$ contained in $\mathbf{Z} G$; equivalently, $\mathfrak{c}=\{x \in \mathbf{Z} G \mid x B \subset \mathbf{Z} G\}$. Moreover, $|G| B \subset \mathfrak{c}[5$, Chapter IX, Corollary 1.2]. Similar remarks hold if we replace $Z$ by the ring of integers in a number field $F$ throughout. It is a theorem of Bass and Murthy [8, Lemma 10.5] that under the above hypotheses, the map $S K_{1}(\mathrm{Z} G, \mathfrak{c}) \rightarrow S K_{1}(B, \mathfrak{c})$ is an isomorphism. ${ }^{2}$ Since $|G| \mathrm{Z} G \subset|G| B \subset \mathfrak{c}, \mathrm{ZG} / \mathrm{c}$ is a finite ring and has trivial $S K_{1}$ [5, Chapter $V$, Corollary 9.2]. We thus deduce from (1.1) that $S K_{1}(\mathrm{ZG})$ is a quotient of $S K_{1}(B, c)$.

Following the decomposition $B=\Pi_{\chi \in S} Z[\bar{\chi}]$, we may write $\mathfrak{c}=\Pi_{\chi \in S^{c}}$; since $S K_{1}$ commutes with finite products, we see that $S K_{1}(\mathrm{Z} G)$, for $G$ finite abelian, is a quotient of $\Pi_{\chi \in S} S K_{1}\left(\mathrm{Z}[\bar{\chi}], c_{\chi}\right)$, and each term of this product is known by Theorem 1.2. Thus $S K_{1}(\mathrm{ZG})$ is a finite abelian group and is precisely the torsion part of $\mathrm{Wh}(G)$ when $G$ is finite abelian. (A more refined analysis shows that any exponent for $G$ is also an exponent for $S K_{1}(\mathrm{Z} G)$.)

\footnotetext{
${ }^{2}$ This property is special to the circumstances described here. In general if $A$ is a subring of $B$ and $I$ is an ideal in both, $S K_{1}(A, I)$ is not necessarily isomorphic to $S K_{1}(B, I)$ [30].
} 
Similar arguments can be used to show that $S K_{1}(A G)$ is finite when $A$ is the ring of integers in an algebraic number field.

Here is an example. Suppose $G$ is an elementary abelian 2-group of rank $k$ (i.e. a product of $k$ cyclic groups of order 2). All characters of $G$ take values in $\{ \pm 1\}$; hence $B \approx Z^{q}$, where $q=2^{k}-1$. Thus $S K_{1}(B, c)=$ $\Pi_{\chi \in S} S K_{1}\left(\mathrm{Z}, \mathrm{c}_{\chi}\right)=0$ (since $\mathrm{Z}$ has a real embedding), and $S K_{1}(\mathrm{Z} G)=0$.

3. Mayer-Vietoris sequences; $K_{2}$. Early workers who tried to exploit the exact sequence $\Pi S K_{1}\left(\mathrm{Z}[\bar{\chi}], \mathrm{c}_{\chi}\right) \rightarrow S K_{1}(\mathrm{Z} G) \rightarrow 0$ to compute Whitehead groups were frustrated by the absence of an extension of this sequence to the left. The algebraic $K_{2}$ functor introduced by Milnor [24] and the resulting MayerVietoris sequences went far towards alleviating this difficulty. I will describe these techniques in some generality before specializing to our particular computational problems.

Suppose

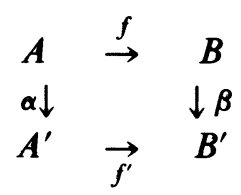

is a commutative diagram of rings and ring homomorphisms with the property that $f$ induces an isomorphism between the ideals $I=\operatorname{ker} \alpha$ and $J=\operatorname{ker} \beta$. Such a diagram is a pullback in the category of rings. Let us assume, in addition:

$$
\begin{aligned}
& \text { For some } i \geqslant 1, \quad K_{i}(A, I) \rightarrow K_{i}(B, J) \text { is onto and } \\
& K_{i-1}(A, I) \rightarrow K_{i-1}(B, J) \text { is an isomorphism. }
\end{aligned}
$$

(In Milnor's original work [5, Chapter VII, §4] he took $i=1$. We are assuming in this general discussion the existence of a "higher" algebraic $K$-theory as, for example, in [27].) Then an easy diagram chase shows that there exists a connecting homomorphism $\partial$ such that the following sequence is exact:

$$
\begin{aligned}
K_{i}(A) & \rightarrow K_{i}(B) \oplus K_{i}\left(A^{\prime}\right) \rightarrow K_{i}\left(B^{\prime}\right) \\
& \stackrel{\partial}{\rightarrow} K_{i-1}(A) \rightarrow K_{i-1}(B) \oplus K_{i-1}\left(A^{\prime}\right) \rightarrow K_{i-1}\left(B^{\prime}\right) .
\end{aligned}
$$

Such a sequence is called a Mayer-Vietoris sequence. It exists for $i=1$ so long as $f^{\prime}$ or $\beta$ is onto. When $i=2$, Milnor showed (3.2) is satisfied provided $f^{\prime}$ and $\beta$ are onto. This leads to an easy proof that $S K_{1}(\mathbf{Z} G)=0$ for a finite cyclic group $G$.

Let us assume, first of all, that $G$ is cyclic of prime order $p$ (we shall see in $\$ 5$ that we can reduce to this case). Then

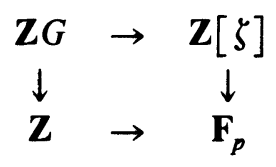

is a pullback with all arrows surjective, where $\zeta$ is a primitive $p$ th root of unity. Here a generator of $G$ is mapped to $\zeta \in \mathbf{Z}[\zeta]$ and to $l \in \mathbf{Z}$, and the 
other maps are reduction mod $1-\zeta$ and $p$. From (3.3) with $i=2$ we deduce the exact sequence

$$
K_{2}\left(\mathbf{F}_{p}\right) \rightarrow S K_{1}(\mathrm{ZG}) \rightarrow S K_{1}(\mathrm{Z}) \oplus S K_{1}(\mathrm{Z}[\zeta])
$$

whose last term is trivial by Theorem 1.2. Since $K_{2}$ of a finite field is also trivial [29], we conclude that $S K_{1}(\mathrm{Z} G)=0$.

A second circumstance in which a Mayer-Vietoris sequence exists for $i=2$ is the conductor situation described above which was first studied by Bass and Murthy. More generally, we have:

(3.4) THEOREM [9]. Let $B=\prod_{i=1}^{n} B_{i}$ be a direct product of (not necessarily commutative) rings and suppose $A \subset B$ is a subring such that each projection of $A$ into a direct factor $B_{i}$ of $B$ is surjective. Let $I$ be any two-sided ideal of $B$ contained in $A$. Then (3.2) holds for the square

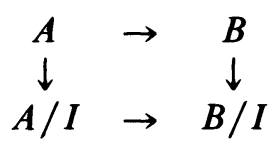

and $i=2$.

4. An algorithm for finite abelian groups. Let me now describe how Theorem 3.4 can be used to calculate, in principle, $S K_{1}(\mathrm{ZG})$ for any finite abelian group $G$. We return to the notation introduced in $\$ 2$; in particular, $B=\Pi_{\chi \in S} \mathrm{Z}[\bar{\chi}]$ is the integral closure in $\mathbf{Q} G$ of $\mathbf{Z} G$, where $S=S_{\mathbf{Q}}(G)$. We choose an ideal $I$ contained in the conductor from $B$ to $\mathbf{Z} G$ and containing a rational integer $(|G| B=I$ is one such choice). We obtain from (3.3) the exact sequence

$$
K_{2}(B) \oplus K_{2}(\mathrm{Z} G / I) \rightarrow K_{2}(B / I) \rightarrow S K_{1}(\mathrm{Z} G) \rightarrow 0 .
$$

This involves noting that $K_{1}$ can be replaced by $S K_{1}$ when the rings are commutative, and applying Theorem 1.2 and the fact that $\mathrm{Z} G / I$ is finite to conclude that

$$
S K_{1}(B)=\Pi S K_{1}(\mathrm{Z}[\bar{\chi}])=0=S K_{1}(\mathrm{Z} G / I) .
$$

Our first task is to analyze the map $K_{2}(B) \rightarrow K_{2}(B / I)$. This may be done componentwise by considering the exact sequences

$$
K_{2}(\mathrm{Z}[\bar{\chi}]) \rightarrow K_{2}\left(\mathrm{Z}[\bar{\chi}] / I_{\chi}\right) \rightarrow S K_{1}\left(\mathrm{Z}[\bar{\chi}], I_{\chi}\right) \rightarrow 0 .
$$

To simplify our task, let us assume henceforth that $G$ is a $p$-group for some prime $p$ (we will shortly show how we may always reduce to this case). The group $K_{2}\left(\mathrm{Z}[\bar{\chi}] / I_{\chi}\right)$ has been computed by Dennis and Stein [10, Theorem 5.1]; combining their calculation with Theorem 1.2 we conclude from (4.2) that $K_{2}(\mathbf{Z}[\bar{\chi}]) \rightarrow K_{2}(\mathbf{Z}[\bar{\chi}] / I)$ is the 0 -map unless $\chi= \pm 1$, in which case the map is onto. Thus we may rewrite (4.1) as follows:

$$
K_{2}(\mathrm{Z} G / I) \stackrel{\varphi}{\rightarrow} \prod_{\chi \in S^{*}} K_{2}\left(\mathrm{Z}[\bar{\chi}] / I_{\chi}\right) \rightarrow S K_{1}(\mathrm{Z} G) \rightarrow 0,
$$

where $S^{*}$ is the subset of $S=S_{\mathrm{Q}}(G)$ different from \pm 1 (of course, $\chi=-1$ can occur only when $p=2$ ) and $\varphi$ is the product map induced by the characters in $S^{*}$. Since all the $K_{2}$ 's which occur in (4.3) are generated by 
Steinberg symbols [28, Theorem 2.13], explicit calculation is often possible. Here is a simple example which indicates the technique.

Let $\zeta$ be a primitive cube root of unity and let $\chi$ be the character of a cyclic group of order 3 which maps a fixed generator to $\zeta$. Let $G$ be the product of 2 cyclic groups of order 3 with generators $\sigma, \tau$, repsectively ( $G$ is an elementary abelian 3-group of rank 2).

Then $\varphi=\left(\chi_{1}, \chi_{2}, \chi_{3}, \chi_{4}\right)$, where $\chi_{1}=\chi \times 1, \chi_{2}=1 \times \chi, \chi_{3}=\chi \times \chi$ and $\chi_{4}=\chi^{-1} \times \chi\left(\right.$ these are the elements of $\left.S^{*}\right)$, and we may take $I_{\chi}=(\zeta-1)^{3}$. By [10, Theorem 3.8(f)] we know that $K_{2}\left(\mathrm{Z}[\zeta] /(\zeta-1)^{3}\right)$ is generated by $s=\left\{\zeta, 1+(\zeta-1)^{2}\right\}$. Also, since each $\chi_{i}$ maps the augmentation ideal, $J$, of $\mathbf{Z} G$ to $(\zeta-1) Z[\zeta]$, it follows that $\chi_{i}\left(J^{3}\right)=0$, and we may replace (4.3) by the exact sequence

$$
K_{2}\left(\mathrm{Z} G /\left(I, J^{3}\right)\right) \stackrel{\varphi}{\rightarrow} K_{2}\left(\mathrm{Z}[\zeta] /(\zeta-1)^{3}\right)^{4} \rightarrow S K_{1}(\mathrm{Z} G) \rightarrow 0 .
$$

Define elements $s_{i} \in K_{2}\left(\mathrm{Z} G /\left(I, J^{3}\right)\right)$ by

$$
\begin{array}{ll}
s_{1}=\left\{\sigma, 1+(\sigma \tau-1)\left(\sigma \tau^{2}-1\right)\right\}, & s_{2}=\left\{\tau, 1+(\sigma \tau-1)\left(\sigma^{2} \tau-1\right)\right\}, \\
s_{3}=\left\{\tau, 1+(\sigma-1)\left(\sigma^{2} \tau^{2}-1\right)\right\}, & s_{4}=\left\{\sigma^{-1}, 1+(\tau-1)\left(\sigma \tau^{2}-1\right)\right\} .
\end{array}
$$

Then $\chi_{i}\left(s_{j}\right)=\delta_{i j} s$ (Kronecker delta), proving $\varphi$ is surjective and $S K_{1}(\mathrm{ZG})=$ 0.

Of course, the above result was known before the advent of the functor $K_{2}$, and our wish is to produce examples where $S K_{1}(\mathrm{Z} G)$ is not trivial. One method, based on [2], and suitable for machine computation, works as follows. Choose a collection of Steinberg symbols $s_{j}$ which generate $K_{2}(\mathrm{ZG} / I)$. Form the matrix with rows indexed by these symbols and columns indexed by $S^{*}$ whose $i j$ th entry is the Steinberg symbol $\chi_{i}\left(s_{j}\right) \in$ $K_{2}\left(Z\left[\bar{x}_{i}\right] / I_{\chi}\right)$. This $K_{2}$ is a cyclic group which may be identified with a subgroup of the roots of unity in $\mathbf{Z}\left[\bar{\chi}_{i}\right]$ by interpreting $\chi_{i}\left(s_{j}\right)$ as a norm residue symbol $[10, \S 4]$. Since explicit formulas for the evaluation of norm residue symbols are known [3], [14], we obtain a relation matrix describing the image of $\varphi$ as a subgroup of $\Pi_{\chi \in S^{*}} K_{2}\left(\mathrm{Z}[\bar{\chi}] / I_{\chi}\right)$ and the order of $S K_{1}(\mathrm{ZG})=$ $\operatorname{coker}(\varphi)$ may be computed. This method has been used by Roy G. Fuller to obtain the following results by machine calculation:

\begin{tabular}{c|c}
$G$ & $S K_{1}(\mathrm{ZG})$ \\
\hline $\mathrm{Z} / p^{2} \times \mathrm{Z} / p^{2}(p=3,5,7)$ & $(\mathrm{Z} / p)^{p-1}$ \\
$\mathrm{Z} / p^{2} \times \mathrm{Z} / p \times \mathrm{Z} / p(p=3,5,7)$ & $(\mathrm{Z} / p)^{p(p-1)}$ \\
$\mathrm{Z} / 27 \times \mathrm{Z} / 9$ & $(\mathrm{Z} / 3)^{4}$ \\
$\mathrm{Z} / 27 \times \mathrm{Z} / 3 \times \mathrm{Z} / 3$ & $(\mathrm{Z} / 3)^{9}$ \\
$\mathrm{Z} / 9 \times \mathrm{Z} / 9 \times \mathrm{Z} / 3$ & $(\mathrm{Z} / 3)^{15} \times(\mathrm{Z} / 9)^{2}$
\end{tabular}

General computations of $S K_{1}(\mathrm{ZG})$ depend, of course, on finding a method for computing the image of $\varphi$. The only general result so far has been for elementary abelian $p$-groups. In that case $\Pi_{\chi \in s^{*}} K_{2}\left(\mathrm{Z}[\bar{\chi}] / I_{\chi}\right)$ is an $F_{p}$-vector space of dimension $\left(p^{k}-1\right) /(p-1)$, where $k$ is the rank of $G$. The 
dimension of $V=\operatorname{image}(\varphi)$ as a subspace of this vector space may be computed by interpreting $V$ as a certain vector subspace of the polynomial functions from the character group of $G$ to $F_{p}$. The result is:

(4.4) THEOREM [2]. Let $p$ be an odd prime and $G$ an elementary abelian p-group of rank $k$. Then $S K_{1}(\mathrm{ZG})$ is an elementary abelian p-group of rank

$$
\frac{p^{k}-1}{p-1}-\left(\begin{array}{c}
p+k-1 \\
p
\end{array}\right)
$$

In particular, $S K_{1}(\mathrm{ZG}) \neq 0$ for $k \geqslant 3$.

Some other results obtained by hand computation are given below [Dennis and Stein, unpublished].

\begin{tabular}{c|c}
$G$ & $S K_{1}(\mathrm{ZG})$ \\
\hline $\mathrm{Z} / 4 \times \mathrm{Z} / 4$ & $\mathrm{Z} / 2$ \\
$\mathrm{Z} / 2 \times \mathrm{Z} / 2 \times \mathrm{Z} / 4$ & $\mathrm{Z} / 2$ \\
$(\mathrm{Z} / 2)^{3} \times \mathrm{Z} / 4$ & $(\mathrm{Z} / 2)^{3} \times \mathrm{Z} / 4$
\end{tabular}

5. Reduction to $p$-groups. The method outlined above for calculating $S K_{1}(\mathrm{Z} G)$ when $G$ is a finite abelian $p$-group applies more generally to the computation of $S K_{1}(\supseteq G)$, where $\mathfrak{D}$ is the ring of integers in any finite Galois extension of $\mathbf{Q}$ in which the prime $p$ does not ramify. We obtain an exact sequence

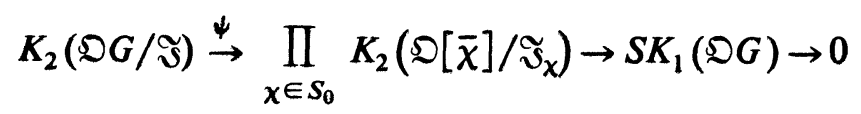

analogous to (4.3), where $S_{0}$ is a certain collection of irreducible characters of $G$ [2]. The norm $N: \mathcal{D} \rightarrow Z$ induces, by extension of scalars, compatible homomorphism $\mathcal{D} G / \mathfrak{\Im} \rightarrow \mathbf{Z} G / I$ and $\mathscr{D}[\bar{\chi}] / \Im_{\bar{x}} \rightarrow \mathbf{Z}[\bar{\chi}] / I_{\chi}$. These, in turn, induce a map of (5.1) to (4.3), which is an isomorphism $S K_{1}(D G) \rightarrow S K_{1}(\mathrm{ZG})$ for $p \neq 2$. (When $p=2, S_{0}$ may be larger than $S^{*}$.) The precise result is as follows.

(5.2) THEOREM. Let $p$ be a prime and $G$ a finite abelian p-group. Let $K_{1} \subset K_{2}$ be a finite Galois extension of number fields with rings of integers $\mathfrak{D}_{1}, \mathfrak{D}_{2}$, respectively, in which $p$ is unramified. Then $S K_{1}\left(\mathfrak{D}_{2} G\right) \approx S K_{1}\left(\mathfrak{N}_{1} G\right)$ in case $p$ is odd, or, when $p=2$, both $\mathfrak{D}_{1}$ and $\mathfrak{D}_{2}$ are totally imaginary or both have real embeddings.

I now want to indicate how Theorem 5.2 can be used to reduce the computation of $S K_{1}(\mathrm{ZG})$ from general finite abelian groups to the case of $p$-groups. For any finite abelian group $G$, let us write $G_{p}$ for its Sylow $p$-subgroup. Thus $G=H \times G_{p}$, where $H$ has order prime to $p$. The integral closure, $C$, of $\mathbf{Z H}$ in $\mathbf{Q} H$ is, by the discussion in \$2, the direct product of $q(H)=q\left(G / G_{p}\right)$ factors of the form $\mathrm{Z}[\zeta]$, where $\zeta$ is a root of unity of order prime to $p$. In particular, each factor $Z[\zeta]$ satisfies the hypothesis of Theorem 5.2 , and it follows that if $p$ is odd, $S K_{1}\left(C\left[G_{p}\right]\right) \approx S K_{1}\left(Z G_{p}\right)^{q\left(G / G_{p}\right)}$. In particular, $S K_{1}\left(C\left[G_{p}\right]\right)$ is a finite abelian $p$-group (cf. §2).

On the other hand, we have the homomorphisms 


$$
\mathbf{Z} G=\mathbf{Z}\left[H \times G_{p}\right] \approx \mathbf{Z}[H]\left[G_{p}\right] \subset C\left[G_{p}\right]
$$

and the induced map $S K_{1}(\mathbf{Z} G) \rightarrow S K_{1}\left(C\left[G_{p}\right]\right)$ kills all torsion other than $p$-torsion, thus inducing a homomorphism

$$
S K_{1}(\mathrm{Z} G)_{p} \stackrel{\alpha}{\rightarrow} S K_{1}\left(C\left[G_{p}\right]\right) .
$$

(5.3) THEOREM [2]. The homomorphism $\alpha$ is an isomorphism for all $p$.

Combining Theorems 5.2 and 5.3, we have

(5.4) TheOREM. Let $G$ be a finite abelian group with Sylow subgroups $\left\{G_{p}\right\}$. Then

$S K_{1}(\mathbf{Z} G) \approx \prod_{\substack{p \text { odd } \\ p|| G \mid}} S K_{1}\left(\mathbf{Z} G_{p}\right)^{q\left(G / G_{p}\right)} \times S K_{1}\left(\mathbf{Z} G_{2}\right) \times S K_{1}\left(\mathbf{Z}\left[\zeta_{3}\right] G_{2}\right)^{q\left(G / G_{2}\right)-1}$,

where $\zeta_{3}$ is a primitive cube root of unity.

6. Induction theorems for finite groups. Let $G$ be a finite abelian group. Since $\mathbf{Q} G$ is semisimple, it follows from the stability theorems for $K_{1}$ [5, Chapter V, Theorem $4.2 \mathrm{ff}$.] that the determinant map from $K_{1}(\mathbf{Q} G)$ to $U(\mathbf{Q} G)$ is an isomorphism, and, therefore, that $\operatorname{ker}\left(K_{1}(\mathbf{Z} G) \rightarrow K_{1}(\mathbf{Q} G)\right)=$ $S K_{1}(\mathrm{Z} G)$. We may thus generalize the definition of $S K_{1}$ to nonabelian finite groups by setting $S K_{1}(\mathbf{Z} G)=\operatorname{ker}\left(K_{1}(\mathbf{Z} G) \rightarrow K_{1}(\mathbf{Q} G)\right.$ ). (There is an alternative method of defining $S K_{1}$ using reduced norms $[4, \S 1]$; we will not need this definition here.) More generally, if $A$ is the ring of integers in an algebraic number field $F$, we set $S K_{1}(A G)=\operatorname{ker}\left(K_{1}(A G) \rightarrow K_{1}(F G)\right)$.

We have already seen in $\$ \$ 1$ and 2 that when $G$ is abelian, the torsion subgroup of $\mathrm{Wh}(A G)$ is precisely $S K_{1}(A G)$. Our next task is to prove Wall's result that this remains true if $G$ is finite, but not necessarily abelian (or, equivalently, to show that $\operatorname{tor}\left(K_{1}(A G)\right)=\operatorname{tor}\left(A^{*}\right) \oplus G^{\mathrm{ab}} \oplus S K_{1}(A G)$ where $A$ is the ring of integers in an algebraic number field and $G$ is finite). To do so will require a brief sketch of the use of induction techniques in algebraic $K$-theory, as developed by Swan, Lam and Dress.

Our starting point is the calculus of induction and restriction for group representations. Let $H$ be a subgroup of a finite group $G$. Any $\mathbf{Z} H$-module $M$ can be made into an induced $\mathbf{Z} G$-module $i_{*}(M)=M \otimes_{\mathbf{Z} H} \mathbf{Z} G$. Conversely, by restricting scalars from $\mathbf{Z} G$ to $\mathbf{Z} H$, any $\mathbf{Z} G$-module $N$ can be made into a $\mathbf{Z} H$-module denoted $i^{*}(N)$. The maps $i_{*}, i^{*}$ are, in fact, functors between the appropriate categories of modules, and are related by the Frobenius reciprocity theorem, which, for our purposes, can be formulated as follows.

Let $G_{\mathrm{Z}}(G)$ (resp. $\left.G_{\mathrm{Z}}(H)\right)$ be the Grothendieck group on the category of all finitely generated $\mathbf{Z} G$ (resp. $\mathbf{Z} H$ )-modules which are $\mathbf{Z}$-projective. Tensor product over $\mathbf{Z}$ induces ring structures on $G_{\mathbf{Z}}(G)$ and $G_{\mathbf{Z}}(H)$, and the Frobenius reciprocity law in this setting states that for $x \in G_{\mathbf{Z}}(G)$, $y \in G_{\mathbf{Z}}(H)$,

$$
i_{*}\left(i^{*}(x) y\right)=x i_{*}(y) .
$$

As usual, similar definitions and results apply when $\mathbf{Z}$ is replaced by a ring of algebraic integers $A$. 
Now suppose $\mathscr{D}$ is some collection of subgroups of $G$, and let $G_{A}(G)_{D}$ be the ideal in $G_{A}(G)$ generated by $i_{*}\left(G_{A}(H)\right)$ for all $H \in \mathscr{D}$.

(6.1) THEOREM [ARTIN]. Let $C$ be the set of all cyclic subgroups of the finite group $G$. Then

$$
|G| G_{\mathbf{Q}}(G) \subset G_{\mathbf{Q}}(G)_{\mathcal{e}} .
$$

We shall often be interested in the collection of hyperelementary subgroups of $G$. A finite group $H$ is said to be $p$-hyperelementary (for some prime $p$ ) if it contains a cyclic normal subgroup of index a power of $p$ (equivalently: if $H$ is the semidirect product $N \rtimes P$ with $N$ normal cyclic and $P$ a $p$-group). Any dihedral group is 2-hyperelementary. All $p$-groups are $p$-hyperelementary.

(6.2) TheOREM [WITt, BERMAN]. Let $\mathcal{H}$ be the collection of p-hyperelementary subgroups of the finite group $G$ for all primes dividing $|G|$. Then $G_{F}(G)=G_{F}(G)_{\mathcal{X}}$ for any algebraic number field $F$.

These theorems were extended by Swan [31], [32].

(6.3) THEOREM. Let $R$ be a Dedekind domain with field of fractions $K$ and let $G$ be a finite group. Suppose $\mathscr{D}$ is a collection of subgroups of $G$. If $n G_{K}(G) \subset$ $G_{K}(G)_{\text {(⿵刀) }}$, then

(i) $n G_{R / \mathfrak{p}}(G) \subset G_{R / \mathfrak{p}}(G)_{\triangleright}$ for all maximal ideals $\mathfrak{p}$ of $R$; and

(ii) $n^{2} G_{R}(G) \subset G_{R}(G)_{\text {Ф. }}$

Swan's work, in turn, was formalized and extended by Lam [21], who showed that whenever theorems such as (6.3) hold for $G_{R}(G)$, they are valid as well for $F(G)$, where $F$ is any functor from finite groups and their monomorphisms to abelian groups which can be given the structure of a "Frobenius module" over $G_{R}$. Examples of such functors are $K_{0}(R G)$, $K_{1}(R G), S K_{1}(R G), \mathrm{Wh}(R G)$.

7. Some computations for nonabelian finite groups. We have seen above that $S K_{1}(\mathrm{Z} G)=0$ when $G$ is cyclic. Hence $S K_{1}(\mathrm{Z} G)_{\mathcal{e}}=0$ for any finite group $G$, where $\mathcal{C}$ is the set of cyclic subgroups of $G$. Using the work of Lam together with Theorems 6.1 and 6.3 , we see that $|G|^{2} S K_{1}(\mathrm{ZG})=0$; i.e. that $S K_{1}(\mathrm{Z} G)$ is a torsion group. Since it is also finitely generated (cf. [5, p. 553, (v)]), we conclude that $S K_{1}(\mathrm{Z} G)$ is finite.

Another corollary of the work of Swan and Lam is:

(7.1) COROLLARY. If $S K_{1}(A H)=0$ for every hyperelementary subgroup $H$ of a finite group $G$, then $S K_{1}(A G)=0$ as well.

By restricting attention to $p$-hyperelementary subgroups for a fixed prime $p$, we can obtain information about the $p$-torsion in $S K_{1}(\mathrm{ZG})$. For example:

(7.2) TheOREM [Swan-Lam]. If $G$ has a cyclic normal Sylow p-subgroup, $S K_{1}(\mathrm{Z} G)$ has no p-torsion.

Let me now indicate, in outline, Wall's argument for proving $S K_{1}(A G)=$ $\operatorname{tor}(\mathrm{Wh}(A G))$. Here $A$ is the ring of integers in an algebraic number field $F$, and we write $K_{\mathrm{r}}^{\prime}(A G)$ for the image of $K_{1}(A G)$ in $K_{1}(F G)$. We have an exact commutative diagram (cf. \&1): 


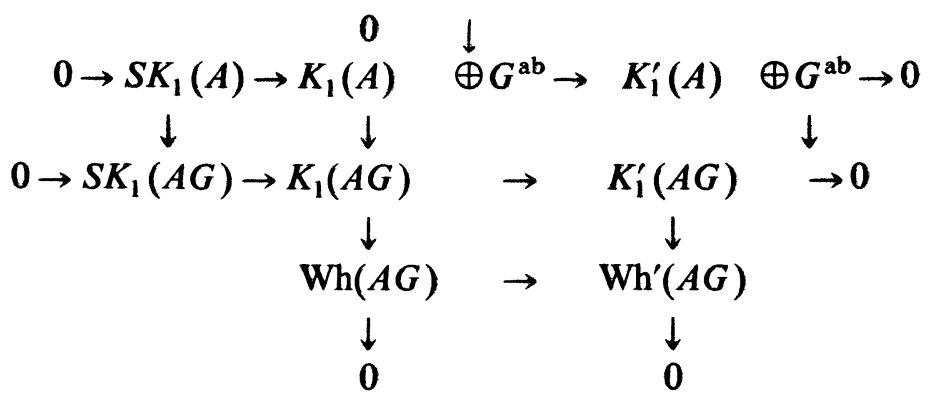

Note that $S K_{1}(A)=0$ by Theorem 1.2. Wall shows that $\mathrm{Wh}^{\prime}(A G)$ is torsionfree, hence that tor $\left(A^{*}\right), G^{\text {ab }}$ and $S K_{1}(A G)$ generate tor $\left(K_{1}(A G)\right)$. Since the diagram remains exact when we restrict to torsion subgroups, it follows that tor $\left.A^{*}\right) \oplus G^{\text {ab }}$ projects isomorphically to $K_{1}^{\prime}(A G)$, which proves that $S K_{1}(A G)$ is a direct summand of $\operatorname{tor}\left(K_{1}(A G)\right)$.

To prove that $\mathrm{Wh}^{\prime}(A G)$ is torsion-free, Wall invokes the induction techniques described above, showing that $\mathrm{Wh}^{\prime}(A G)$ is $p$-torsion free if $\mathrm{Wh}^{\prime}(A H)$ is $p$-torsion free for every $p$-hyperelementary subgroup $H$ of $G$. Next he proves that if $K$ is a normal $l$-subgroup of $H$ for some prime $l \neq p, \mathrm{Wh}^{\prime}(A H)$ is $p$-torsion free if $\mathrm{Wh}^{\prime}(A[H / K])$ is. This allows him to reduce to the case when $H$ is a $p$-group, for which direct arguments are possible.

Theorem 7.2 has been used to prove that $S K_{1}\left(Z D_{2 p}\right)=0$, where $D_{2 p}$ is the dihedral group of order $2 p, p$ an odd prime. Let $I$ be the ideal of $\mathbf{Z} D_{2 p}$ generated by all $g-1$, where $g$ lies in the Sylow $p$-subgroup of $D_{2 p}$. Then $S K_{1}\left(\mathbf{Z} D_{2 p} / I\right)=0$, and it follows that $S K_{1}\left(\mathbf{Z} D_{2 p}\right)$ is a quotient of $S K_{1}\left(\mathrm{Z} D_{2 p}, I\right)$. Direct computation shows that this relative group is a $p$-group. Since Theorem 7.2 implies that $S K_{1}\left(Z D_{2 p}\right)$ has no $p$-torsion, it must be trivial. These results are due to Lam [21] for $p=3$ and to Keating [18] and Obayashi [25].

Similar arguments have been used by Keating to show $S K_{1}(\mathrm{Z} G)=0$ for any metacyclic group $G$ containing a normal subgroup $H$ of prime index $s$ relatively prime to $|H|$. He has also noted [19] that these methods prove the triviality of $S K_{1}(\mathrm{Z} G)$ if the normal subgroup $H$ has order $p,|G / H|$ does not divide $p-1$ and $G / H$ embeds in the automorphism group of $H$.

Along slightly different lines, Keating [17] and Obayashi [26] have proved $S K_{1}(\mathbf{Z D})=0$ when $D$ is a dihedral 2-group. Keating produces an order $D$ in $Q D$ and an ideal $I \subset \mathcal{D}$ such that the usual map $S K_{1}(\mathcal{D}, I) \rightarrow S K_{1}(\mathcal{D})$ factors as

$$
S K_{1}(\mathcal{Q}, I) \stackrel{\alpha}{\rightarrow} S K_{1}(\mathrm{Z} D) \stackrel{\beta}{\rightarrow} S K_{1}(\mathcal{Q}),
$$

with $\alpha$ onto and $\beta$ injective. Explicit computation then shows that $\beta \alpha$ is the 0 -map. The same technique gives a similar result for semidihedral 2-groups [Keating, unpublished].

Finally, Magurn [22] has generalized the results of Keating and Obayashi to show $S K_{1}(\mathrm{ZD})=0$ for all dihedral groups $D$. His method uses MayerVietoris sequences reminiscent of $\$ 3$ to proceed inductively from the cases $|D|=2^{r}$ and $|D|=2 p$. Since these dihedral groups are hyperelementary, Magurn is able to apply his result in conjunction with Corollary 7.1 to prove: 
(7.3) THEOREM. Let $S_{n}$ be the nth symmetric group. $S K_{1}\left(Z_{S_{n}}\right)=0$ for $n=4$, 5,6 . More generally, $S K_{1}(\mathrm{ZG})=0$ for any permutation group $G$ of degree $\leqslant 6$.

Similarly, $S K_{1}(\mathrm{Z} G)=0$ when $G$ is the binary tetrahedral or icosahedral group. The same is true for the binary octahedral group, provided that $S K_{1}(\mathrm{Z} H)=0$ for the generalized quaternion group $H$ of order 16 . Whether this is, in fact, true, remains an open question.

\section{REFERENCES}

1. R. C. Alperin, R. K. Dennis and M. R. Stein, The nontriviality of $S K_{1}\left(Z_{\pi}\right)$, Proc. Conf. on Orders, Group Rings and Related Topics, Lecture Notes in Math., vol. 353, Springer-Verlag, Berlin and New York, 1973, pp. 1-7.

2.,$S K_{1}$ of finite abelian groups (to appear).

3. E. Artin and H. Hasse, Die beiden Ergänzungssätze zum Reziprozitätsgesetz der $l^{n}$-ten Potenzreste im Körper der $l^{n}$-ten Einheitswurzeln, Abh. Math. Sem. Univ. Hamburg 6 (1928), 146-162.

4. H. Bass, The Dirichlet unit theorem, induced characters, and Whitehead groups of finite groups, Topology 4 (1966), 391-410.

5. __ Algebraic K-theory, Benjamin, New York, 1968.

6. I I $S K_{1}(Z \Pi)=0$ for $\Pi$ a finite abelian group, Proc. Conf. on Orders, Group Rings and Related Topics, Lecture Notes in Math., vol. 353, Springer-Verlag, Berlin and New York, 1973, p. 84.

7. H. Bass, J. Milnor and J.-P. Serre, Solution of the congruence subgroup problem for $\mathbf{S L}_{\boldsymbol{n}}$ $(n \geqslant 3)$ and $\mathrm{Sp}_{2 n}(n \geqslant 2)$, Inst. Hautes Étude Sci. Publ. Math. No. 33 (1967), 59-137.

8. H. Bass and M. P. Murthy, Grothendieck groups and Picard groups of abelian group rings, Ann. of Math. (2) 86 (1967), 16-73.

9. R. K. Dennis, The computation of Whitehead groups, Notes of a course at Universität Bielefeld, 1973.

10. R. K. Dennis and M. R. Stein, $K_{2}$ of discrete valuation rings, Advances in Math. 18 (1975), 182-238.

11. A. W. M. Dress, Contributions to the theory of induced representations, Lecture Notes in Math., vol. 342, Springer-Verlag, Berlin, 1973, pp. 183-240.

12. A. Fröhlich, M. E. Keating and S. M. J. Wilson, The class groups of quaternion and dihedral 2-groups, Mathematika 21 (1974), 64-71.

13. G. Higman, The units of group rings, Proc. London Math. Soc. (2) 46 (1940), 231-248.

14. K. Iwasawa, On explicit formulas for the norm residue symbol, J. Math. Soc. Japan 20 (1968), 151-165.

15. M. E. Keating, Class groups of metacyclic groups of order p'q, Mathematika 21 (1974), 90-95.

16. On the K-theory of the quaternion group, Mathematika 20 (1973), 59-62.

17. Whitehead groups of dihedral groups (private communication).

18. __ Whitehead groups of some metacyclic groups and orders, J. Algebra 22 (1972), 332-349.

19. _ Postscript to Journal of Algebra paper (private communication).

20. W Whitehead torsion for the quaternion group of order $4 p$ (holograph).

21. T.-Y. Lam, Induction theorems for Grothendieck groups and Whitehead groups of finite groups, Ann. Sci. Ecole Norm. Sup. (4) 1 (1968), 91-148.

22. B. Magurn, $S K_{1}$ of dihedral groups, J. Algebra (to appear).

23. J. Milnor, Whitehead torsion, Bull. Amer. Math. Soc. 72 (1966), 358-426.

24. __ Introduction to algebraic K-theory, Ann. of Math. Studies, No. 72, Princeton Univ. Press, Princeton, N.J., 1971.

25. T. Obayashi; On the Whitehead group of the dihedral group of order $2 p$, Osaka J. Math. 8 (1971), 291-297.

26. _ The Whitehead groups of dihedral 2-groups, J. Pure Appl. Algebra 3 (1973), 59-71.

27. D. Quillen, Higher algebraic K-theory. I, Lecture Notes in Math., vol. 341, Springer-Verlag, Berlin, 1973, pp. 85-147. 
28. M. R. Stein, Surjective stability in dimension 0 for $K_{2}$ and related functors, Trans. Amer. Math. Soc. 178 (1973), 165-191.

29. R. Steinberg, Générateurs, rélations et revetements de groupes algébriques, Colloq. Théorie des Groupes Algébriques (Bruxelles, 1962), Librairie Universitaire, Louvain; Gauthier-Villars, Paris, 1962, pp. 113-127.

30. R. G. Swan, Excision in algebraic K-theory, J. Pure Appl. Algebra 1 (1971), 221-252.

31. __ Induced representations and projective modules, Ann. of Math. (2) 71 (1960), $552-578$.

32. _. The Grothendieck group of a finite group, Topology 2 (1963), 85-110.

33. C. T. C. Wall, Norms of units in group rings, Proc. London Math. Soc. (3) 29 (1974), 593-632.

Department of Mathematics, NorthWestern University, Evanston, IllinOIS 60201 